Article

\title{
Active Switching of Extremely High-Q Fano Resonances Using Vanadium Oxide-Implanted Terahertz Metamaterials
}

\author{
Jing Ma ${ }^{1}$, Zhi-Hang Wang ${ }^{2}$, Huan Liu ${ }^{1, *}$, Ya-Xian Fan ${ }^{1, *}$ and Zhi-Yong Tao ${ }^{1, *(D)}$ \\ 1 Guangxi Key Laboratory of Wireless Wideband Communication and Signal Processing, Guilin University of \\ Electronic Technology, Guilin 541004, China; jingma900526@163.com \\ 2 Key Lab of In-fiber Integrated Optics, Ministry Education of China, Harbin Engineering University, \\ Harbin 150001, China; wangzhihang@hrbeu.edu.cn \\ * Correspondence: huanliu@guet.edu.cn (H.L.); yxfan@guet.edu.cn (Y.-X.F.); zytao@guet.edu.cn (Z.-Y.T.)
}

Received: 7 December 2019; Accepted: 31 December 2019; Published: 2 January 2020

\begin{abstract}
In this paper, we demonstrate an active switching of extremely high Q-factor Fano resonances using vanadium oxide $\left(\mathrm{VO}_{2}\right)$-implanted $\mathrm{THz}$ asymmetric double $\mathrm{C}$-shaped metamaterial (MM) structures. The simulation results indicate the highly temperature-sensitive nature of the double Fano resonances that can be switched at very low external thermal pumping $\left(68^{\circ} \mathrm{C}\right)$, which is only slightly higher than room temperature. We employ the surface current and electric field distributions of the structure to analyze the physical mechanism of the observed switching behavior in the thermally excited Fano MMs. More importantly, by optimizing the asymmetric parameter (offset length), the linewidth of the Fano resonance can reach only $0.015 \mathrm{THz}$ and the Q-factor is as high as 98, which is one order of magnitude higher than that of the traditional MMs. The findings of this work would enable a thermally-induced high-Q Fano resonance MMs for ultra-sensitive sensors, modulators, low threshold switching in metadevices.
\end{abstract}

Keywords: terahertz; metamaterials; vanadium dioxide; thermal control; switching; Fano resonances

\section{Introduction}

The fifth-generation mobile communication technology (5G) has been initially realized and gradually began to be commercialized in the world. Therefore, how to accelerate the basic research of the next generation mobile communication technology, namely the sixth-generation (6G) [1], is the most concerned problem of current researchers. The mainstream frequency band of $5 \mathrm{G}$ is $3 \mathrm{GHz}-6$ $\mathrm{GHz}$, belonging to millimeter wave frequency range. However, to improve the transmission rate, communication bandwidth and information capacity of the system, it has been reported that the working frequency band of $6 \mathrm{G}$ network may be elevated to the higher terahertz $(\mathrm{THz})$ region. Due to its high frequency, short pulse, strong penetrability, and low energy, $\mathrm{THz}$ wave has been widely used in communication, imaging, sensing and other fields. It is worth mentioning that $\mathrm{THz}$ spectrum has not been widely occupied so far, which is very suitable for future wireless communications [2]. Thus, in order to effectively promote the development and application of 6G technology in the future, researchers are committed to developing new THz radiation sources, detectors, and functional devices [3].

At present, $\mathrm{THz}$ functional devices are still relatively scarce, and the main limiting factor is the lack of natural reaction materials. It is generally known that the polar insulating material is transparent to $\mathrm{THz}$ waves while the metal electron completely shields $\mathrm{THz}$ waves, so the most natural materials hardly respond to $\mathrm{THz}$ radiation. The emergence of artificial composite materials called metamaterials (MMs) with periodically or randomly arranged subwavelength resonance unit has greatly solved this problem [4]. Different from natural materials, the dielectric constant and permeability of MMs 
can be arbitrarily changed by adjusting the micro structure units and their arrangements, so as to achieve effective control of $\mathrm{THz}$ waves, such as amplitude, phase, polarization, and wavelength. The unique physical properties of MMs usually originate from the resonance effect of structural elements. Resonance response mainly has three essential characteristics including resonant frequency, modulation depth (or resonant intensity) and Q-factor. To achieve sharp resonance with high Q-factor is the most effective strategy to greatly improve the performance of metadevices. It is of great significance in the fields of hypersensitive biochemical sensors [5], high-performance filtering [6], plasmon lasers [7], and slow light devices [8]. For example, using high Q-factor resonance with very narrow linewidth can realize ultra-sensitive real-time chemical and biomolecular detection, and resonance with large modulation depth will improve the detection sensitivity $[9,10]$.

However, due to the limitation of ohmic loss and radiation loss, the conventional metal MM resonance usually has a lower Q-factor (less than 10), which seriously limits the performance of metadevices. To achieve a high Q-factor resonance response, it is necessary to suppress both radiation loss and ohmic loss. In the THz band, most precious metals have very high conductivity, which can be regarded as perfect conductors. Thus, the ohmic loss of the MMs constructed by them is generally very low while the radiation loss is dominant. To obtain the Fano resonance with high Q-factor, it is very important to minimize the radiation loss of MMs. The theoretical and experimental results have confirmed that by introducing the asymmetric structure into the MMs, the high Q-factor Fano resonance response can be achieved [11,12]. Fano resonance is a linear asymmetric resonance produced by the elimination or interference of a wide continuous spectrum and a narrow discrete resonance [13]. Its physical mechanism is to drive the anti-phase oscillation current to decrease the radiation loss.

It is worth noting that, in addition to the study of MMs with high Q-factor and modulation depth, considering the tunability and flexibility of metadevices in various practical applications, such as optical switches, optical sensors, and ultra-sensitivity spectroscopy, regulation of high Q-factor Fano resonance is also urgently needed. Researchers have focused on tunable THz MMs with high Q-factor and modulation depth, and proposed different types of asymmetric Fano resonance structures, such as dipole bars [14], metal strip [15], ring structure [16], and planar defective [17]. The resonance frequency, modulation depth and linewidth of the Fano resonance can be tuned to a limited extent by adjusting the geometric parameters of the MMs structures in a passive way.

Recently, a small number of research findings have preliminarily demonstrated that if the novel active materials are integrated into MMs, such as semiconductors [18] and graphene [19-21] with the assist of external stimulation, the Fano resonances will achieve precise active control at additional level, which is of great value to practical applications of metadevices. As a kind of active material, $\mathrm{VO}_{2}$ has a temperature-sensitive insulator-metal transition (IMT) characteristic. Under the external thermal excitation, its conductivity can be rapidly increased by $4-5$ orders of magnitude and is only one order of magnitude lower than that of gold [22,23]. Therefore, $\mathrm{VO}_{2}$ has also attracted great attention of researchers in development of high-performance active tunable $\mathrm{THz} \mathrm{MM}$ devices, such as resonators [24], modulator [25], polarization controller [26], optical switch [27,28], beam controller [29], and absorber [30].

In this paper, we demonstrate an active switching of extremely high-Q Fano resonances using $\mathrm{VO}_{2}$-implanted $\mathrm{THz}$ asymmetric double $\mathrm{C}$-shaped $\mathrm{MM}$ structure. The simulation results show that the sharp Fano resonance with the high Q-factor can be switched using very low external thermal excitation $\left(68^{\circ} \mathrm{C}\right)$, which is only slightly higher than the room temperature. The simulated surface current and electric field distributions of MM structures are employed to describe the resonance mechanism of the thermally excited Fano MMs. By optimizing the asymmetric parameters (offset length), the Q-factor of Fano resonance can achieve 98, which is one order of magnitude higher than that of the traditional MMs. This kind of high Q-factor MMs have tremendous potential in the fields of ultra-sensitive sensors, ultra-narrow band filters, and optical switches. 


\section{2. $\mathrm{VO}_{2}$ Materials}

To describe the properties of $\mathrm{VO}_{2}$ thin films in incomplete phase transition, we need to use the effective medium theory (EMT). At present, the most commonly used are Maxwell-Garnett EMT and Bruggeman EMT. Because the metal particles in composite media are non-uniform distribution and the volume fraction of metal components is more than $20 \%$ during phase transition, Maxwell-Garnett EMT is not suitable for this condition [31]. In THz band, the IMT characteristics of $\mathrm{VO}_{2}$ can be described by Bruggeman EMT. The dielectric function $\varepsilon_{C}$ can be expressed as

$$
\varepsilon_{C}=\frac{1}{4}\left\{\begin{array}{c}
\varepsilon_{D}\left(2-3 f_{v}\right)+\varepsilon_{M}\left(3 f_{v}-1\right) \\
+\sqrt{\left[\varepsilon_{D}\left(2-3 f_{v}\right)+\varepsilon_{M}\left(3 f_{v}-1\right)\right]^{2}+8 \varepsilon_{D} \varepsilon_{M}}
\end{array}\right\}
$$

where $f_{v}$ is the volume fraction of the metal region, $\varepsilon_{D}$ and $\varepsilon_{M}$ are dielectric functions of $\mathrm{VO}_{2}$ thin films in insulation and metal phase, respectively.

The functional relationship between the volume fraction $f_{v}$ and temperature $T$ follows Boltzmann function,

$$
f_{v}=f_{\max }\left(1-\frac{1}{1+\exp \left[\left(T-T_{0}\right) / \Delta T\right]}\right)
$$

where $T_{0}$ is the phase transition temperature, with heating and cooling points $68^{\circ} \mathrm{C}$ and $64{ }^{\circ} \mathrm{C}$, respectively; $\Delta T=2{ }^{\circ} \mathrm{C}$ is the transition width, and $f_{\max }$ is the maximum volume fraction of the metal component in $\mathrm{VO}_{2}$ at the highest temperature. Experimental results show that $\Delta T=2{ }^{\circ} \mathrm{C}$ and $f_{\max }=$ 0.95 [23].

For the insulator component in $\mathrm{VO}_{2}$, it can be regarded as a dielectric with dielectric constant $\varepsilon_{D}$ $=9$ [23]. The dielectric function $\varepsilon_{M}$ of metal components in $\mathrm{VO}_{2}$ can be determined by Drude model. However, the traditional Drude model cannot accurately describe the dielectric function of $\mathrm{VO}_{2}$ in IMT process. Therefore, to make the Drude model better represent IMT characteristic of $\mathrm{VO}_{2}$, a simple generalization was derived based on the impulse response approach and Poisson statistics [32,33], and the negative parameter $b$ is introduced $[23,34]$,

$$
\varepsilon_{m}=\varepsilon_{\infty}-\frac{\omega_{p}^{2}}{\omega^{2}+i \omega \Gamma}\left(1+\frac{i b \Gamma}{\omega+i \Gamma}\right)
$$

where $\omega=2 \pi f$ is the circular frequency of THz wave, $\varepsilon_{\infty}=\varepsilon_{D}=9$ is the high-frequency limit dielectric constant of $\mathrm{VO}_{2}, \omega_{p}$ is the plasma frequency, which is expressed as,

$$
\omega_{p}=\sqrt{N e^{2} / \varepsilon_{0} m^{*}}
$$

where $N=1.3 \times 10^{22} \mathrm{~cm}^{-3}$ is the carrier concentration in the medium, $m^{*}=2 m_{\mathrm{e}}$ is the effective mass, $m_{\mathrm{e}}=9.1094 \times 10^{-31} \mathrm{~kg}$ is the electronic mass and $\varepsilon_{0}$ is the vacuum dielectric constant. $\Gamma=1 / \tau$ is the scattering rate, where $\tau=2.27 \times 10^{-15} \mathrm{~s}$ is the carrier collision time.

The complex permittivity can be expressed as a summation of real and imaginary parts,

$$
\varepsilon(\omega)=\varepsilon_{1}(\omega)+\frac{4 \pi i}{\omega} \sigma_{1}(\omega)
$$

where $\varepsilon_{1}(\omega)$ is the real dielectric function, and $\sigma_{1}(\omega)$ is the frequency dependent conductivity. The expression for the conductivity and real dielectric function of $\mathrm{VO}_{2}$ thin films corresponding to different temperatures in the phase transition process can be acquired by comparing Equation (1) of EMT, the Equation (2) of Boltzmann's function, Equations (3) and (4) of Drude's model, and Equation (5) of complex permittivity,

$$
\sigma_{1}(\omega)=\frac{n e^{2} \Gamma / m}{\left(\Gamma^{2}+\omega^{2}\right)}\left[1+\frac{\mathrm{b}\left(\Gamma^{2}-\omega^{2}\right)}{\left(\Gamma^{2}+\omega^{2}\right)}\right]
$$




$$
\varepsilon_{1}(\omega)=\varepsilon_{\infty}-\frac{\omega_{p}^{2}}{\left(\Gamma^{2}+\omega^{2}\right)}\left[1+\frac{2 \mathrm{~b} \Gamma^{2}}{\left(\Gamma^{2}+\omega^{2}\right)}\right]
$$

Around $1.0 \mathrm{THz}, \mathrm{VO}_{2}$ film conductivity in the metal phase $\approx 27000 \mathrm{~S} / \mathrm{m}$, and $b=-0.6579$, from previous experimental data $[22,23,35]$. Figure 1 shows conductivities in heating and cooling processes around $\mathrm{VO}_{2}$ critical temperature (red dot and blue dash-dot curves, respectively) are close to experimental data. The effect of temperature on the real part $\varepsilon_{1}(\omega)$ of the dielectric constant of metal phase $\mathrm{VO}_{2}$ is negligible.



Figure 1. Conductivity of $\mathrm{VO}_{2}$ during phase transition. The heating and cooling processes agree well with experimental data [23].

\section{3. $\mathrm{VO}_{2}$-Implanted $\mathrm{THz}$ Metamaterials}

Figure 2 presents the graphical representation of the $\mathrm{VO}_{2}$-implanted $\mathrm{THz}$ asymmetric double $\mathrm{C}$-shaped MM sample under the thermal pump and $\mathrm{THz}$ pulse irradiation. The detailed geometry dimensions of the unit cell are shown in the upper right inset. The yellow area is symmetrical double C-shaped resonance structure composed of gold, which can be used to generate a dipole resonance. To excite the high Q-factor Fano resonance and realize the active tunable function, we introduce a phase-change $\mathrm{VO}_{2}$ rectangular array (purple area) into the MMs, breaking the symmetry of the original structure. Asymmetric parameter (offset length $a$ ) is used to describe the extent of structural asymmetry, which refers to the distance between the center of the MM unit structure $l_{x}$ and the center of the gap $d$, as shown in the red dotted line. The thickness of gold and $\mathrm{VO}_{2}$ is $1 \mu \mathrm{m}$. To eliminate the interference of the substrate to the $\mathrm{THz}$ spectrum, the polyimide with high $\mathrm{THz}$ wave transmittance is selected as the substrate material and the thickness is set to $20 \mu \mathrm{m}$. We preliminarily predict that $\mathrm{VO}_{2}$ in the insulating phase at room temperature is transparent to incident $\mathrm{THz}$ wave and the designed metadevice behaves as a dipole resonance. However, when the metadevice is excited by external heat, the $\mathrm{VO}_{2}$ film embedded in the MMs plays a quasi metallic role, the symmetry of the structure is broken, and then it will show the Fano resonance characteristic. Thus, using the external thermal excitation, it is easy to realize the mutual conversion of different resonance modes and active switching of the Fano resonance.

The Fano resonance mode switching behavior of the proposed $\mathrm{VO}_{2}$-implanted $\mathrm{THz}$ asymmetric MM can be characterized by using three-dimensional full wave electromagnetic simulation software COMSOL Multiphysics based on the finite element method. The periodic boundary conditions are set in the $x$ and $y$ direction and the perfect matched layers are assigned along the $z$ direction. The incident THz pulse propagates perpendicularly to the $x-y$ plane and the electric field component is along the $y$-axis direction. Because the linewidth of the Fano resonance excited by designed MM structure is very narrow, in order to improve the frequency resolution of $\mathrm{THz}$ spectrum, the simulated frequency step is set to $0.005 \mathrm{THz}$, and the ultra-fine grid is adopted. The dielectric constant of polyimide substrate is 
2.25 and the dielectric dispersion of gold material is formulated by Drude model, $\varepsilon(\omega)=1-\omega_{p}^{2} /\left(\omega^{2}+\right.$ $i \gamma \omega)$, where the resonance frequency of plasma is $\omega_{p}=1.37 \times 10^{16} \mathrm{rad} / \mathrm{s}$, the damping coefficient is $\gamma=4.08 \times 10^{13} \mathrm{rad} / \mathrm{s}$ [36]. In addition, considering the asymmetric line shape of Fano resonance, to fully characterize the resonance characteristics of the metadevice, we define the modulation depth as $M_{d}=\left[\left(T_{\max }-T_{\min }\right) / T_{\max } \times 100 \%\right]$, where $T_{\max }$ and $T_{\min }$ represent the maximum and minimum transmittances at the resonance frequency, respectively. From the energy point of view, the $\mathrm{Q}$-factor is defined as $Q=2 \pi f_{0} \times P_{S} / P_{L}=f_{0} / \Delta f$, where $f_{0}$ is the central frequency of the resonance, $P_{S}$ and $P_{L}$ represent the stored and dissipated energy, respectively, and $\Delta f$ is the full width at half maximum (FWHM) of resonance.

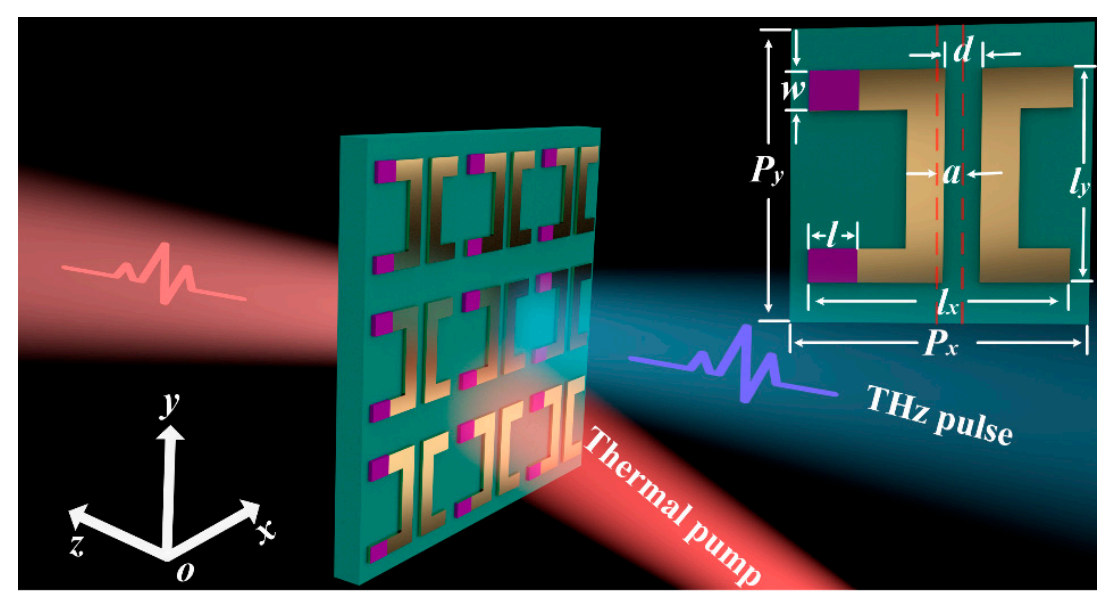

Figure 2. Graphical representation of the $\mathrm{VO}_{2}$-implanted terahertz $(\mathrm{THz})$ asymmetric double C-shaped metamaterial (MM) sample under the thermal pump and $\mathrm{THz}$ pulse irradiation. The upper right inset shows the detailed geometry dimensions of the unit cell with period length $P x=P y=180 \mu \mathrm{m}$, resonator length $l x=140 \mu \mathrm{m}$ and $l y=120 \mu \mathrm{m}$, resonance linewidth $w=15 \mu \mathrm{m}, \mathrm{VO}_{2}$ rectangular strip length $l=32 \mu \mathrm{m}$ and gap $d=15 \mu \mathrm{m}$, (asymmetric parameter) offset length $a=16 \mu \mathrm{m}$

In order to verify the experimental feasibility of the proposed active switching of extremely high Q-factor Fano resonances, the fabrication process and characterization method of the device are described in detail. $\mathrm{VO}_{2}$ films can be successfully prepared by pulsed laser deposition (PLD), sputtering or sol-gel method (SGM). The quality and uniformity of the prepared $\mathrm{VO}_{2}$ films can be accurately characterized by high resolution X-ray diffraction (XRD), Raman spectroscopy and Fourier transform infrared spectroscopy (FTIR). By means of photolithography and a micro-fabrication process, the $\mathrm{VO}_{2}$-implanted $\mathrm{THz} \mathrm{MMs}$ structure can be easily obtained. First, the $\mathrm{VO}_{2}$ layer is patterned via reactive ion etching after photolithography. Gold films are then deposited on the samples by electron beam evaporation following the second photolithography. Eventually, the samples are fabricated using the lift-off process [37].

To accurately characterize the switching behavior of the metadevice, we can employ traditional THz time-domain spectral (THz-TDS) based on femtosecond laser [38]. The measured sample should be placed in a temperature control box so that its surface temperature can be adjusted in real time. At the same time, the area of sample is much larger than that of the $\mathrm{THz}$ beam spot, which can ensure the validity of the measurement. The $\mathrm{THz}$ time-domain signal and Fourier spectrum information of air reference and sample can be extracted, then the transmission spectrum corresponding different frequencies can be obtained. To eliminate the influence of water vapor noise on the experimental results, the whole experimental measurement must be carried out in nitrogen atmosphere. The thin and transparent polymer substrate in the MM structure will greatly reduce the interference of the substrate to the measured $\mathrm{THz}$ spectrum. It should be emphasized that the frequency resolution of THz-TDS will have a great influence on the measured resonance intensity and Q-factor. Therefore, 
based on the above analysis, we preliminarily predict that as long as the frequency resolution of system is high enough, the results of experiments and simulations will be in good agreement [39].

\section{Active Switching of Fano Resonances}

To excite the strong Fano resonance effect, we set the offset length to $16 \mu \mathrm{m}$, and then obtain the MM structure with large asymmetry ratio. Figure 3a describes the transmission response of the proposed $\mathrm{VO}_{2}$-implanted $\mathrm{MM}$ structure for heating process. The blue curve represents the transmission spectrum of the metadevice at low temperature $\left(45^{\circ} \mathrm{C}\right)$. We only observe a broad eigen resonance mode at $0.700 \mathrm{THz}$ excited by dipole resonance (acts as a bright mode), which has a wide bandwidth of 0.385 $\mathrm{THz}$ and low $\mathrm{Q}$-factor of 3 . When the $\mathrm{VO}_{2}$-implanted $\mathrm{MM}$ is radiated by thermal pump of $58^{\circ} \mathrm{C}$, the double Fano resonance modes are excited on both sides of the eigen resonance, including Fano 1 at 0.515 $\mathrm{THz}$ and Fano 2 at $1.160 \mathrm{THz}$ (behaves as a dark mode). Upon further enhancement of the pumping heat, Fano resonance dips attenuates gradually, and a small red shift occurs at the resonance frequency, it is completely switched when the pump power is increased to $68^{\circ} \mathrm{C}$. The pumping temperature of 70 ${ }^{\circ} \mathrm{C}$ will not affect the Fano resonance any more. The observed Fano resonance in the thermally excited $\mathrm{VO}_{2}$-implanted MMs is mainly due to the destructive interference caused by the asymmetry of the structure. Its physical mechanism is the resonance interaction between the wide dipole mode and the sharp discrete mode.
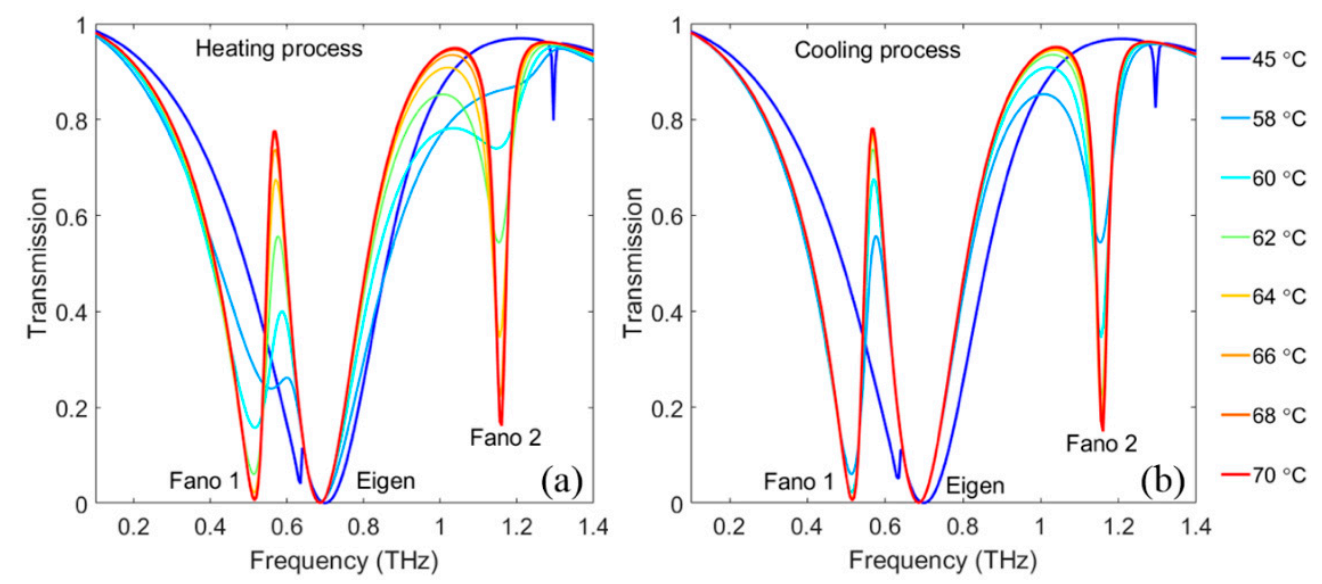

Figure 3. Simulated $\mathrm{THz}$ transmission spectra of the proposed $\mathrm{VO}_{2}$-implanted $\mathrm{MM}$ structure corresponding to different temperatures, showing the active modulation of the two Fano resonances at $0.515 \mathrm{THz}$ (Fano 1) and $1.160 \mathrm{THz}$ (Fano 2): (a) heating process; (b) cooling process.

In order to characterize the resonance and tunable performance of the proposed metadevice, we calculated the Q-factor and modulation depth of the excited Fano resonances. It is exciting that although the calculated Q-factor of Fano 1 is only 5, but its modulation depth is as high as $99 \%$. More importantly, compared with the intrinsic resonance mode, the resonance response of Fano 2 is obviously improved, the Q-factor is 33, which is much higher than that of the known MMs, simultaneously, and the modulation depth can reach $83 \%$. This kind of MM with large modulation depth and high Q-factor has great potential in ultra-sensitive sensing and ultra-narrow band filtering applications. The excited strong Fano resonance can be completely switched-off by cooling the metadevice, but this process is not reversible. The simulation results in Figure $3 b$ presents that when the external excitation temperature decreases from $70{ }^{\circ} \mathrm{C}$ to $64^{\circ} \mathrm{C}$, the strength of the observed Fano resonance has not been significantly weakened. Upon further reducing the pump power, Fano dips in the transmission spectrum abates gradually and it completely disappears when temperature drops to $45^{\circ} \mathrm{C}$. Therefore, compared with the thermal excitation process, it is difficult to switch off the Fano resonance using the cooling power.

The simulation results shown in Figure 3 have clearly characterized the excellent switching performance of the metadevice. To describe the continuously tunable process more vividly under the 
external thermal excitation, the two-dimensional $\mathrm{THz}$ transmission spectrum changing with frequency and temperature has been simulated and is shown in Figure 4. For the heating process described in Figure $4 \mathrm{a}$, we can see that the weak Fano 1 can be excited at around $60^{\circ} \mathrm{C}$. With the increasing temperature, Fano 2 is also excited and the resonance intensity is gradually strengthened. When the metadevice is heated to $70{ }^{\circ} \mathrm{C}$, the intensity of Fano resonance reaches the maximum. Simultaneously, the simulated results given in Figure $4 \mathrm{~b}$ describe the continuous tunability of Fano resonance better by using the cooling process. The initial cooling does not effectively affect the Fano resonance of both Fano 1 and Fano 2 until the temperature dropped to about $60^{\circ} \mathrm{C}$. The Fano resonances are gradually switched off with the decrease of external temperature. When the temperature dropped to $55^{\circ} \mathrm{C}$, the Fano resonance completely disappears. The simulated two-dimensional plot clearly shows that compared with the heating process, it is not easy to switch off the Fano resonance by cooling the structure. That is to say, the switch process of Fano resonance realized by utilizing the temperature control is not reversible. Herein, it should be highlighted that the active switching of Fano resonance can be realized by using very low heat pump closest to the room temperature, which is difficult to obtain in other tunable Fano devices. The simulation results demonstrate the highly temperature-sensitive nature of the sharp Fano resonances to the external thermal stimulus.
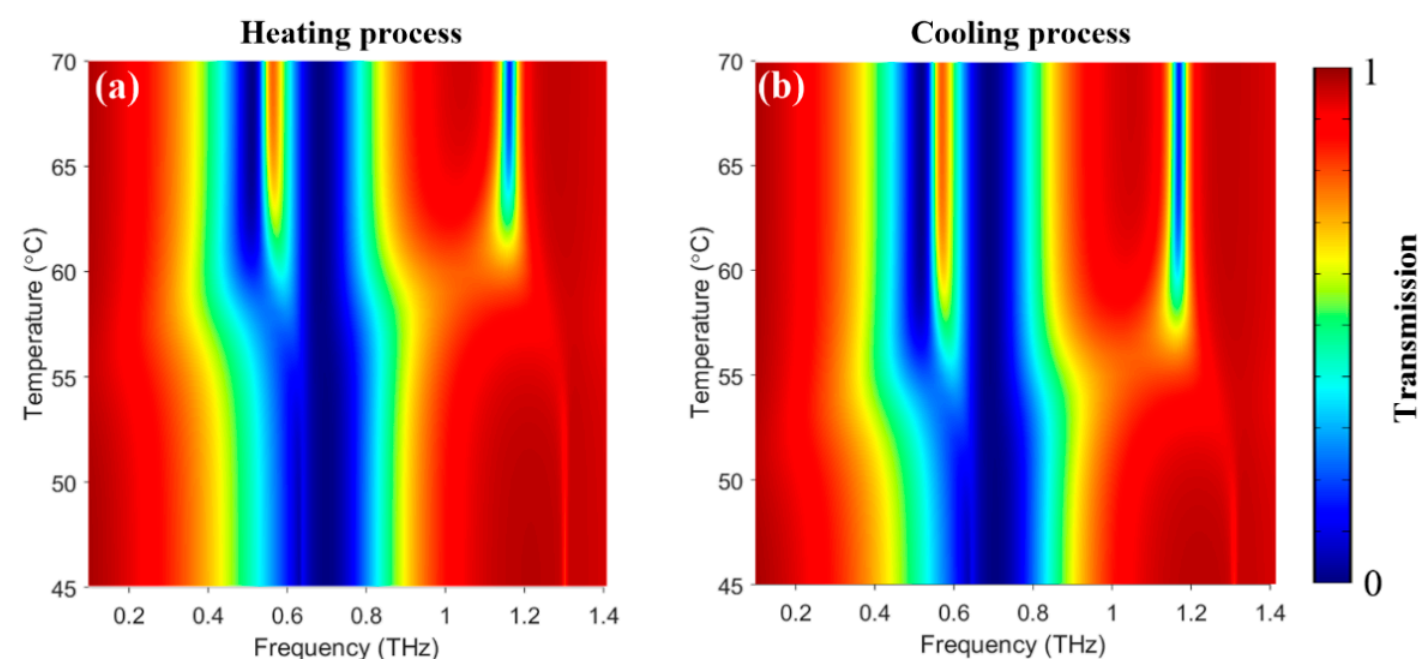

Figure 4. Two-dimensional graph of simulated $\mathrm{THz}$ transmission spectra corresponding to frequency and temperature of the proposed $\mathrm{VO}_{2}$-implanted $\mathrm{MM}$ structures, describing the continuously tunable process of Fano 1 and Fano 2: (a) heating process; (b) cooling process.

We preliminarily predict that the observed active switching of sharp Fano resonance is closely associated with the change in the conductivity of $\mathrm{VO}_{2}$ film embedded $\mathrm{MM}$ structures. For the heating process, at low temperature, $\mathrm{VO}_{2}$ behaves as insulator with low conductivity and is transparent to incident $\mathrm{THz}$ waves. Thus, the proposed MM structure is equivalent to the symmetrical double-C shaped periodic array composed of gold, which can generate the eigen resonance mode excited by the dipole resonance. As the increasing pump temperature, the number of carriers in $\mathrm{VO}_{2}$ material increases, which results in the sharp increase in conductivity. Hence, the phase changed $\mathrm{VO}_{2}$ rectangular strips in the designed MMs play a role of quasi-metals with a skin depth around $1 \mu \mathrm{m}$ at $1.0 \mathrm{THz}$. This change of $\mathrm{VO}_{2}$ destroys the symmetry of the double-C shaped $\mathrm{MM}$ structures and excites the double Fano resonances. However, Fano resonance induced by cooling process is completely different. The main reason for this phenomenon is that the IMT temperatures of $\mathrm{VO}_{2}$ in the process of heating and cooling are quite different, which are $68^{\circ} \mathrm{C}$ and $64{ }^{\circ} \mathrm{C}$, respectively, leading to different regulation behavior of the metadevice.

In addition, there is an obvious physical phenomenon in Figures 3 and 4 that cannot be ignored, that is, at low temperature $\left(45^{\circ} \mathrm{C}\right)$, we have not only observed an eigen resonance mode in the whole 
spectrum, but also two sharp micro resonance dips with low transmission at $0.635 \mathrm{THz}$ and $1.295 \mathrm{THz}$. It can be explained as follows: Although $\mathrm{VO}_{2}$ behaves as an insulator at low temperature, it is still not completely transparent to $\mathrm{THz}$ waves and has a small loss, which destroys the symmetry of the structure a little and produces the two small Fano resonance dips. From another point of view, this physical phenomenon also further confirms the physical mechanism of Fano resonance excited by the asymmetry of the MM structure.

\section{Analysis of Physical Mechanism}

In order to clarify the basic properties and internal physical mechanism of the thermal controlled Fano resonance in detail, we simulated the surface current and electric field distributions at the corresponding resonant frequency for the low $\left(45^{\circ} \mathrm{C}\right)$ and high $\left(70{ }^{\circ} \mathrm{C}\right)$ temperatures, as shown in Figure 5. The surface current in the asymmetric $\mathrm{VO}_{2}$-implanted $\mathrm{MM}$ structures can be decomposed into a series of current oscillation units, which is equivalent to the electromagnetic waves radiated from the electric dipole to the far field. The physical mechanism of resonance can be well explained by the theory of electromagnetic scattering and dipole radiation [40]. For eigen resonance mode of $0.700 \mathrm{THz}$ at low temperature shown in Figure 5a, the surface current and electric field intensity are mainly concentrated in the relatively small C-shaped structure on the right side and the induced current direction is the same as the incident $\mathrm{THz}$ electric field along the $y$ axis. The electromagnetic field radiated by the corresponding electric dipole produces constructive interference, which results in high radiation loss, and creates the dipole resonance with a low Q-factor.
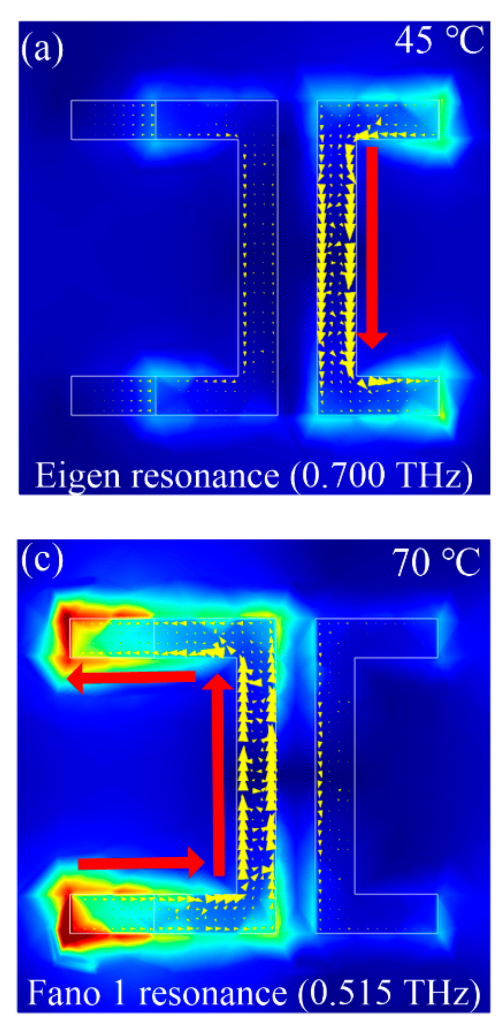
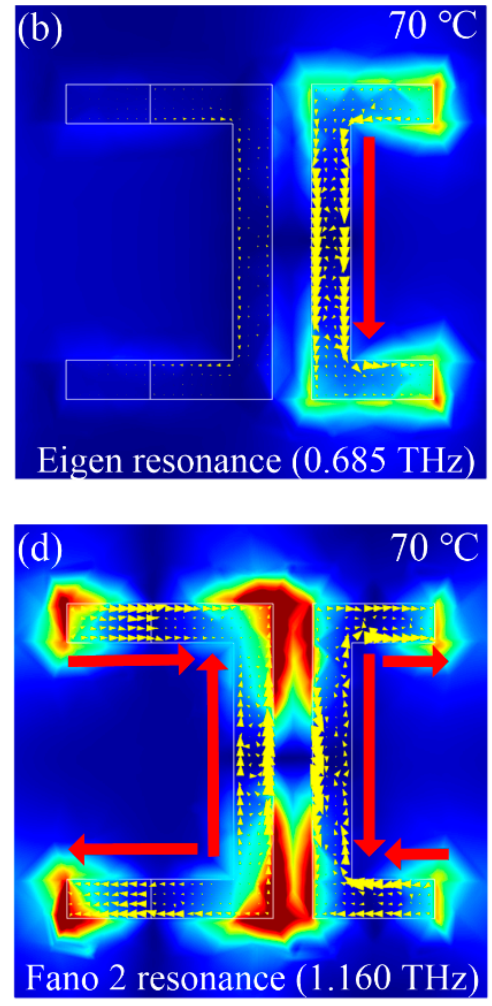

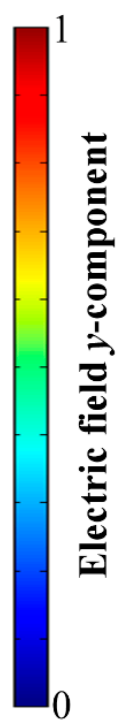

Figure 5. Surface current and electric filed distributions of the proposed $\mathrm{VO}_{2}$-implanted $\mathrm{MM}$ structures at the frequencies of Fano resonances for the low and high temperatures. (a) Eigen resonance $(0.700 \mathrm{THz})$ at $45^{\circ} \mathrm{C}$. (b) Eigen resonance $(0.685 \mathrm{THz}),(\mathbf{c})$ Fano $1(0.515 \mathrm{THz})$, and (d)_Fano $2(1.160 \mathrm{THz})$ at $70{ }^{\circ} \mathrm{C}$.

For the three resonance modes excited at high temperature, the corresponding surface current and electric field distributions are shown in Figure $5 b-d$. The eigen resonance $(0.685 \mathrm{THz})$ shown in Figure $5 \mathrm{~b}$ displays the same characteristic of the dipole resonance, except for a slight increase in structure coupled electric field energy. For the Fano 1 induced by the high temperature metal phase 
$\mathrm{VO}_{2}$ shown in Figure $5 \mathrm{c}$, the surface electric field and current are mainly concentrated in the relatively large $\mathrm{C}$-shaped structure on left side. The current in the $x$ direction always anti-phase oscillates (i.e., pointing in the opposite direction). Therefore, the equivalent dipole moment produced by the oscillating current along the $x$ direction can almost cancel each other, which greatly reduce the radiation damping of the MM structures and promote the formation of a high Q-factor resonance.

For the extremely sharp Fano 2 in Figure 5d, it is clear that there are multiple current and electric field oscillation nodes in the MM structures. The phase of current oscillation is both opposite in the $x$ and $y$ directions, resulting in the cancellation of the electric dipole moment. So, the radiation loss is significantly suppressed. More importantly, the diffraction coupling effect plays an indispensable role in the creation of a high Q-factor Fano resonance [39]. The simulated surface electric field distributions corresponding to Fano 2 clearly show that the ultra-strong local current and electric field are coupled in the asymmetry $\mathrm{VO}_{2}$-implanted $\mathrm{MM}$ structures. The coupled electric field energy of Fano 2 is two orders of magnitude higher than that of the eigen resonance shown in Figure $5 b$.

According to the theory of diffraction coupling [41], the resonant frequency of lattice surface mode is $f_{L S M}=c \sqrt{i^{2}+j^{2}} / n p$, where $c$ represents the speed of light in vacuum and $n$ is the refractive index of substrate, $p$ is the lattice constant of the MM array (the period length), and $i, j$ is the order of lattice mode. The calculated resonant frequency of the first-order lattice surface mode corresponding to the asymmetry $\mathrm{VO}_{2}$-implanted $\mathrm{MM}$ structures at high temperature is $1.112 \mathrm{THz}$, which is very close to the response frequency of Fano 2. Hence, THz photon energy can be strongly restricted in the MMs through diffraction coupling, which greatly enhances the energy stored in the subwavelength resonant unit. Based on the above analysis, the incident $\mathrm{THz}$ photon energy is strongly bounded in the MMs and the radiation damping is suppressed by the cancellation interference of electric dipole, which leads to the extremely high Q-factor of Fano 2.

To further optimize the Q-factor and modulation depth of the Fano resonances, we study the influence of asymmetric parameters on the spectral response. Figure $6 \mathrm{a}, \mathrm{b}$ show one- and two-dimensional graphs of the simulated $\mathrm{THz}$ transmission spectra corresponding to different asymmetric parameters (offset length $a$ ), respectively. It can be seen that when the offset length is $0 \mu \mathrm{m}$, i.e. the symmetrical double C-shaped structure is presented, we can only observe the eigen resonance excited by dipole resonance in the whole transmission spectrum, as the dark blue curve shown in Figure 6a. As expected, the increasing offset length breaks the symmetry of the structure, inducing the resonances of Fano 1 and Fano 2 on both sides of the eigen resonance. Interestingly, the increasing offset length makes the eigen resonance mode move to the high frequency range (the blue shift), while the excited Fano 1 and Fano 2 shift to the low frequency (the red shift). The resonance linewidth is gradually widened and the resonance dip is significantly attenuated.

(a)

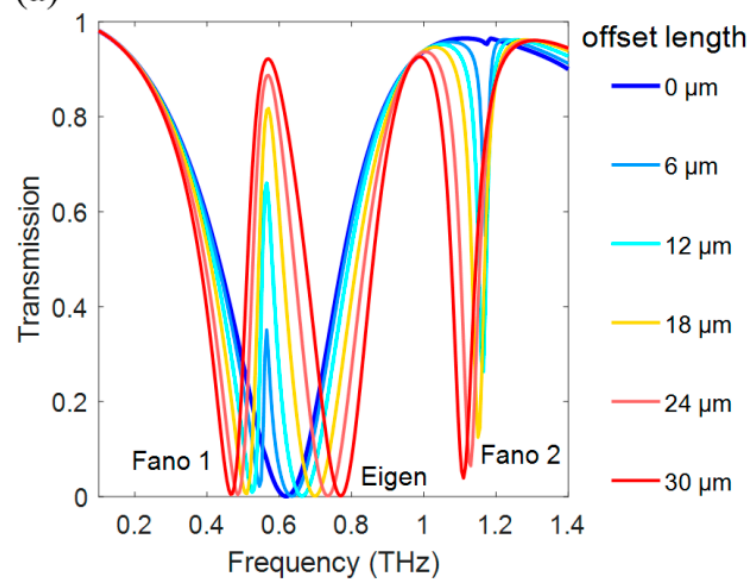

(b)



Figure 6. Transmission spectra of the $\mathrm{VO}_{2}$-implanted $\mathrm{MM}$ structures for different asymmetric parameters (offset length) at high temperature $\left(70^{\circ} \mathrm{C}\right)$ : (a) one-and (b) two-dimensional graphs. 
Using the surface current and electric field distributions in Figure 5, we can easily understand the influence of offset length on the resonant spectrum. For the eigen resonance, the electric field intensity is mainly coupled to the C-shaped structure on the right side, while the corresponding electric field intensities of Fano 1 and Fano 2 are mainly distributed on the right-side C-shaped structure. Therefore, the increase of offset length results in the extension of the left geometrical length and the reduction of the right side. The arm length is inversely proportional to the response frequency in MMs, which produces different resonant modes [42].

We analyze the relationship between the Q-factor and modulation depth of Fano 1 and Fano 2 with the offset length, as shown in Figure 7a,b. Although obtaining the maximum Q-factor and the modulation depth at the same time is the pursuit of many device applications, there is a significant trade-off between the Q-factor and the modulation depth of these two Fano resonances. For Fano 1, as the offset length changes from $3 \mu \mathrm{m}$ to $30 \mu \mathrm{m}$, the Q-factor drops sharply from 33 to 4 while the modulation depth increases rapidly from 63.5\% to 99.4\%, as shown in Figure 7a. For Fano 2, as long as a small offset length is introduced, Fano 2 will appear immediately and the Q-factor is up to 98 while the modulation depth is only 16.5\%, as shown in Figure $7 \mathrm{~b}$. It should be noted that Fano 1 and Fano 2 are dark modes that cannot be obtained in symmetry structure and they only appear when symmetry of structure is destroyed. Changing the asymmetric parameter will not lead to the essential change of these two resonances, but will greatly affect their Q-factor and modulation depth.

(a)

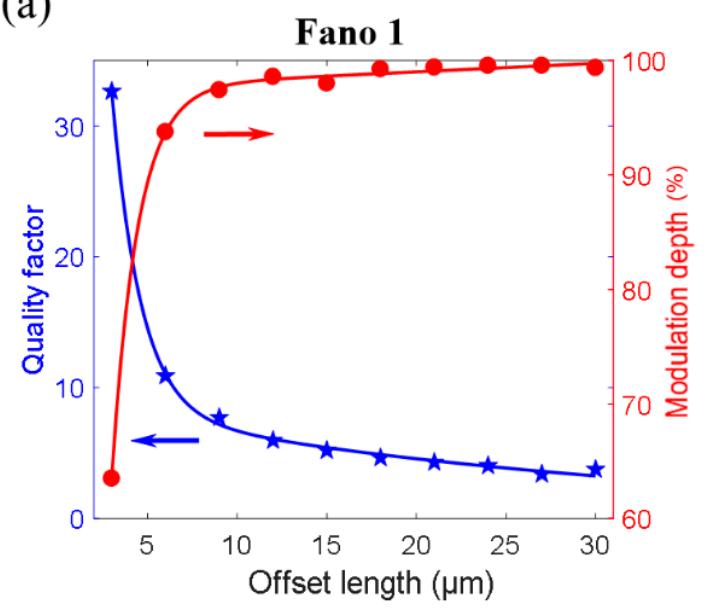

(b)

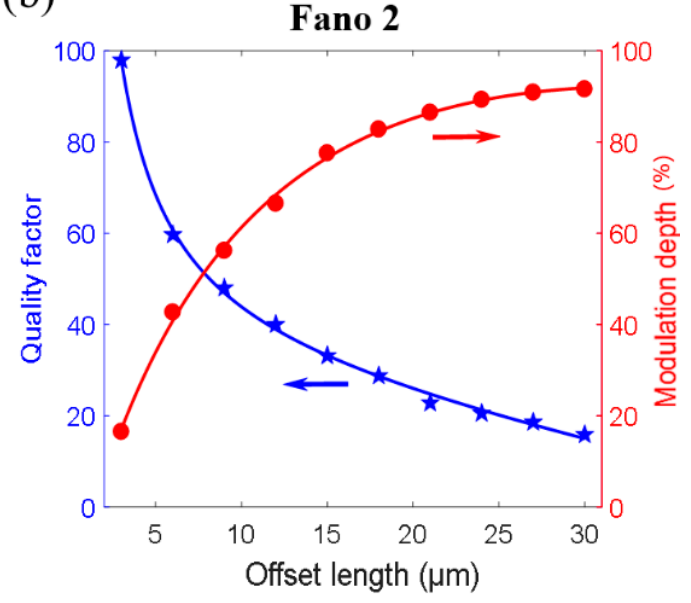

Figure 7. Q-factor and modulation depth of the two Fano resonances for the varying asymmetric parameter (offset length) of the $\mathrm{VO}_{2}$-implanted $\mathrm{MM}$ structures at high temperature $\left(70{ }^{\circ} \mathrm{C}\right)$ : (a) Fano 1 and (b) Fano 2.

To reflect the superior performance of the proposed active switching, we provide a clear comparison between the parameters of designed metadevice with others previously reported experimentally and numerically among the literature $[18,19,43,44]$, including THz metamaterial type, type of switching, resonance frequency, $\mathrm{Q}$ factor and modulation depth, the results are shown in Table 1 . It can be seen that compared with the $\mathrm{VO}_{2}$ implanted $\mathrm{THz} \mathrm{MMs}$, the graphene, photosensitive silicon and MEMS MMs can also be employed to realize the switching behaviors of Fano resonance. However, the tunable range of the constitutive parameters of materials is limited, which leads to the low modulation depth. For the switch control type, the electronic control switch needs to design complex electrode structure in the MM structure, which greatly increases the difficulty of device processing. We can easily realize the switching function by light excitation, but this increases the cost of metadevice to a large extent. It is exciting that the thermal control switch designed by us can realize the active regulation of two Fano resonance modes $(0.52 \mathrm{THz}$ and $1.16 \mathrm{THz})$ at the same time. The control temperature is close to room temperature, and the Q-factor and modulation depth can also be kept at a higher level, which is difficult to achieve in other active switches. 
Table 1. The comparison between the predicted performance with others previously reported.

\begin{tabular}{ccccc}
\hline THz Metamaterial Type & Switching Control Type & $\begin{array}{c}\text { Fano Resonance } \\
\text { Frequency (THz) }\end{array}$ & Q-Factor & $\begin{array}{c}\text { Modulation } \\
\text { Depth (\%) }\end{array}$ \\
\hline Hybrid $\mathrm{VO}_{2}$ & Thermal & 0.52 and 1.16 & 98 & 99 \\
Silicon-implanted [18] & Light & 0.55 & 7 & 84 \\
Graphene [19] & Voltage & 1.43 & 33 & 70 \\
MEMS [43] & Voltage & 0.56 & 380 & 70 \\
Hybrid graphene [44] & Voltage & 0.89 & 9 & 38 \\
\hline
\end{tabular}

\section{Conclusions}

In conclusion, we have demonstrated an active switching of extremely high-Q Fano resonances by utilizing the $\mathrm{VO}_{2}$-implanted $\mathrm{THz}$ symmetry-broken double $\mathrm{C}$-shaped MMs. Due to the IMT characteristic of $\mathrm{VO}_{2}$, the active switching of Fano resonances can be realized at a very low external heat pump $\left(68^{\circ} \mathrm{C}\right)$, which is only a little higher than the room temperature. The simulation results confirmed the highly temperature-sensitivity nature of the Fano resonances of the proposed metadevice, which is difficult to be achieved in other devices. At the same time, using the cooling process, we can completely switch off the excited Fano resonances. It should be highlighted that the regulation processes of the Fano resonances base on the means of heating and cooling are quite different, and it is more difficult to switch off the Fano resonances by using the cooling process. In order to clarify the basic properties and internal physical mechanism of the thermal controlled Fano resonances, we simulated the surface current and electric field distributions at the corresponding resonant frequencies for the low $\left(45^{\circ} \mathrm{C}\right)$ and high $\left(70^{\circ} \mathrm{C}\right)$ temperatures. To further optimize the Q-factor and modulation depth of the Fano resonances at high temperature $\left(70^{\circ} \mathrm{C}\right)$, we have investigated the influence of asymmetric parameter (the offset length) on the spectral response, which show a significant compromise between the Q-factor and the modulation depth. The resonant linewidth of Fano 2 at $1.160 \mathrm{THz}$ is $0.015 \mathrm{THz}$ and the extremely high Q-factor is 98 , which is one order of magnitude higher than that of traditional MMs. For the technologically difficult $\mathrm{THz}$ region, the designed thermally-induced Fano resonance MMs would open up avenues for advanced design and realization of high-performance photonic devices, such as ultra-sensitive sensors, modulators, and low threshold switching.

Author Contributions: Investigation, J.M. and Z.-H.W.; Methodology, J.M., Z.-H.W. and H.L.; Software, J.M. and Z.-H.W.; Supervision, Y.-X.F. and Z.-Y.T.; Writing—original draft, J.M., Z.-H.W. and H.L.; Writing-review and editing, Y.-X.F. and Z.-Y.T. All authors have read and agreed to the published version of the manuscript.

Funding: This research was funded by Dean Project of Guangxi Key Laboratory of Wireless Wideband Communication and Signal Processing.

Conflicts of Interest: The authors declare no conflict of interest.

\section{References}

1. Klaus, D.; Hendrik, B. 6G vision and requirements: Is there any need for beyond 5G. IEEE Veh. Technol. Mag. 2018, 13, 72-80.

2. Nagatsuma, T.; Ducournau, G.; Renaud, C. Advances in terahertz communication accelerated by photonics. Nat. Photonics 2016, 10, 371-379. [CrossRef]

3. Tonouchi, M. Cutting-edge terahertz technology. Nat. Photonics 2007, 1, 97-105. [CrossRef]

4. Zheludev, N.I.; Kivshar, Y.S. From metamaterials to metadevices. Nat. Mater. 2012, 11, 917-924. [CrossRef] [PubMed]

5. Singh, R.W.; Cao, I.; Al-Naib, L.; Cong, W.; Zhang, W. Ultrasensitive terahertz sensing with high-Q Fano resonances in metasurfaces. Appl. Phys. Lett. 2014, 105, 171101. [CrossRef]

6. Paul, O.; Beigang, R.; Rahm, M. Highly selective terahertz bandpass filters based on trapped mode excitation. Opt. Express 2009, 17, 18590-18595. [CrossRef] [PubMed]

7. Zheludev, N.I.S.; Prosvirnin, N.; Fedotov, V. Lasing spaser. Nat. Photonics 2008, 2, 351-354. [CrossRef] 
8. Totsuka, K.; Kobayashi, N.; Tomita, M. Slow light in coupled-resonator-induced transparency. Phys. Rev. Lett. 2007, 98, 213904. [CrossRef]

9. Wu, C.; Arju, N.; Kelp, G.J.; Fan, A.J.; Dominguez, E.; Gonzales, E.; Tutuc, I.; Shvets, G. Spectrally selective chiral silicon metasurfaces based on infrared Fano resonances. Nat. Commun. 2014, 5, 3892. [CrossRef]

10. Barth, A.; Zscherp, C. What vibrations tell about proteins. Q. Rev. Biophys. 2002, 35, 369-430. [CrossRef]

11. Fedotov, V.A.; Rose, M.S.L.; Zheludev, N.I. Sharp trapped-mode resonances in planar metamaterials with a broken structural symmetry. Phys. Rev. Lett. 2007, 99, 147401. [CrossRef] [PubMed]

12. Cao, W.; Singh, R.; Al-Naib, I.; He, M.; Taylor, A.J.; Zhang, W. Low-loss ultra-high-Q dark mode plasmonic Fano metamaterials. Opt. Lett. 2012, 37, 3366-3368. [CrossRef] [PubMed]

13. Fano, U. Effects of configuration interaction on intensities and phase shifts. Phys. Rev. 1961, 124, 1866-1878. [CrossRef]

14. Lim, W.X.; Singh, R. Universal behaviour of high-Q Fano resonances in metamaterials: Terahertz to near-infrared regime. Nano Converg. 2018, 5, 5. [CrossRef]

15. Qin, X.; Guang, X.D.; Wang, B.X.; Wei, Q.H. High-Q Fano resonance in terahertz frequency based on an asymmetric metamaterial resonator. Nano. Res. Lett. 2018, 13, 294.

16. Lan, S.; Qing, L.Z.; Wan, L.; Ge, L.; Jian, H.S.; Cun, L.Z. Effect of the broken symmetry on the electromagnetic properties in the terahertz ring resonators. Opt. Commun. 2019, 445, 136-141.

17. Chen, L.; Xu, N.; Singh, L.; Cui, T.J.; Singh, R.J.; Zhu, Y.M.; Zhang, W.L. Defect-induced Fano resonances in corrugated plasmonic metamaterials. Adv. Opt. Mater. 2017, 5, 1600960. [CrossRef]

18. Manjappa, M.; Srivastava, Y.K.; Cong, L.; Al-Naib, I.; Singh, R.J. Active photoswitching of sharp Fano resonances in THz metadevices. Adv. Mater. 2017, 29, 1603355. [CrossRef]

19. He, X.Y.; Lin, F.T.; Liu, F.; Shi, W.Z. Tunable high Q-factor terahertz complementary graphene metamaterial. Nanotechnology 2018, 29, 485205. [CrossRef]

20. Zhen, D.Y.; Lina, Q.; Peng, Z. Generation of tunable double Fano resonances by plasmon hybridization in graphene-metal metamaterial. Appl. Phys. Express 2018, 11, 072001.

21. Wang, X.; Liu, G.; Xia, S.; Meng, H.; Shang, X.J.; He, P.B.; Xiang, Z. Dynamically tunable Fano resonance based on graphene metamaterials. IEEE Photon. Technol. Lett. 2018, 30, 2147-2150. [CrossRef]

22. Mandal, P.; Speck, A.; Ko, C.; Ramanathan, S. Terahertz spectroscopy studies on epitaxial vanadium dioxide thin films across the metal-insulator transition. Opt. Lett. 2011, 36, 1927-1929. [CrossRef] [PubMed]

23. Jepsen, P.U.; Fischer, B.M.; Thoman, A.; Helm, H.; Suh, J.Y.; Lopez, R.; Haglund, R.F. Metal-insulator phase transition in a $\mathrm{VO}_{2}$ thin film observed with terahertz spectroscopy. Phys. Rev. B 2006, 74, 205103. [CrossRef]

24. Wang, S.; Kang, L.; Werner, D.H. Hybrid resonators and highly tunable terahertz metamaterials enabled by vanadium dioxide $\left(\mathrm{VO}_{2}\right)$. Sci. Rep. 2017, 7, 4326. [CrossRef]

25. Zhou, G.; Dai, P.; Wu, J. Broadband and high modulation-depth THz modulator using low bias controlled $\mathrm{VO}_{2}$-integrated metasurface. Opt. Express 2017, 25, 17322-17328. [CrossRef]

26. Wang, S.; Kang, L.; Werner, D.H. Active terahertz chiral metamaterials based on phase transition of vanadium dioxide $\left(\mathrm{VO}_{2}\right)$. Sci. Rep. 2018, 8, 189. [CrossRef]

27. Ding, F.; Zhong, S.; Bozhevolnyi, S.I. Vanadium dioxide integrated metasurfaces with switchable functionalities at terahertz frequencies. Adv. Opt. Mater. 2018, 6, 1701204. [CrossRef]

28. Wang, D.; Zhang, L.; Gu, Y.; Mehmood, M.Q.; Gong, Y.; Srivastava, A.; Jian, L.; Venkatesan, T.; Qiu, C.W.; Hong, M. Switchable ultrathin quarter-wave plate in terahertz using active phase-change metasurface. Sci. Rep. 2015, 5, 15020. [CrossRef]

29. Hashemi, M.R.M.; Yang, S.H.; Wang, T.; Sepúlveda, N.; Jarrahi, M. Electronically-controlled beam-steering through vanadium dioxide metasurfaces. Sci. Rep. 2016, 6, 35439. [CrossRef]

30. Song, Z.; Wang, K.; Li, J.; Liu, Q.H. Broadband tunable terahertz absorber based on vanadium dioxide metamaterials. Opt. Express 2018, 26, 7148-7154. [CrossRef]

31. Skryabin, I.L.; Radchik, A.V.; Moses, P.; Smith, G.B. The consistent application of Maxwell-Garnett effective medium theory to anisotropic composites. Appl. Phys. Lett. 1997, 70, 2221-2223. [CrossRef]

32. Cocker, T.L.; Titova, L.V.; Fourmaux, S.; Bandulet, H.C.; Brassard, D.; Kieffer, J.C. Terahertz conductivity of the metal-insulator transition in a nanogranular $\mathrm{VO}_{2}$ film. Appl. Phys. Lett. 2010, 97, 221905. [CrossRef]

33. Smith, N.V. Classical generalization of the Drude formula for the optical conductivity. Phys. Rev. B 2001, 64, 155106. [CrossRef] 
34. Turner, G.M.; Beard, M.C.; Schmuttenmaer, C.A. Carrier localization and cooling in dye-sensitized nanocrystalline titanium dioxide. J. Phys. Chem. B 2002, 106, 11716-11719. [CrossRef]

35. Wen, Q.Y.; Zhang, H.W.; Yang, Q.H.; Xie, Y.S.; Chen, K.; Liu, Y.L. Terahertz metamaterials with $\mathrm{VO}_{2}$ cut-wires for thermal tenability. Appl. Phys. Lett. 2010, 97, 021111. [CrossRef]

36. Ordal, M.A.; Long, L.L.; Bell, R.J.; Bell, S.E.; Bell, R.R.; Alexander, R.W.; Ward, C.A. Optical properties of the metals $\mathrm{Al}, \mathrm{Co}, \mathrm{Cu}, \mathrm{Au}, \mathrm{Fe}, \mathrm{Pb}, \mathrm{Ni}, \mathrm{Pd}, \mathrm{Pt}, \mathrm{Ag}, \mathrm{Ti}$ and $\mathrm{W}$ in the infrared and far infrared. Appl. Opt. 1983, 22, 1099-1120. [CrossRef]

37. Zhang, C.H.; Zhou, G.C.; Wu, J.B.; Tang, Y.H.; Wen, Q.Y.; Li, S.X.; Han, J.G.; Jin, B.B.; Chen, J.; Wu, P.H. Active control of terahertz waves using vanadium-dioxide-embedded metamaterials. Phys. Rev. Appl. 2019, 11, 054016. [CrossRef]

38. Exter, M.V.; Fattinger, C.; Grischkowsky, D. Terahertz time-domain spectroscopy of water vapor. Opt. Lett. 1989, 14, 1128-1130. [CrossRef]

39. Yang, S.; Tang, C.; Liu, Z.; Wang, B.; Wang, C.; Li, J.J.; Wang, L.; Gu, C.Z. Simultaneous excitation of extremely high-Q-factor trapped and octupolar modes in terahertz metamaterials. Opt. Express 2017, 25, 15938. [CrossRef]

40. Yang, S.; Liu, Z.; Xia, X.; Yiwen, E.; Tang, C.; Wang, Y.; Li, J.; Wang, L.; Gu, C. Excitation of ultrasharp trapped-mode resonances in mirror-symmetric metamaterials. Phys. Rev. B 2016, 93, 235407. [CrossRef]

41. Manjappa, M.; Srivastava, Y.K.; Singh, R. Lattice-induced transparency in planar metamaterials. Phys. Rev. $B$ 2016, 94, 161103. [CrossRef]

42. Okamoto, T.; Otsuka, T.; Sato, S.; Fukuta, T.; Haraguchi, M. Dependence of LC resonance wavelength on size of silver split-ring resonator fabricated by nanosphere lithography. Opt. Express 2012, 20, 24059-24067. [CrossRef] [PubMed]

43. Manjappa, M.; Pitchappa, P.; Singh, N.; Wang, N.; Zheludev, N.I.; Lee, C.; Singh, R. Reconfigurable MEMS Fano metasurfaces with multiple-input-output states for logic operations at terahertz frequencies. Nat. Commun. 2018, 9, 4056. [CrossRef] [PubMed]

44. Xiao, S.; Wang, T.; Jiang, X.; Yan, X.; Cheng, L.; Wang, B.; Xu, C. Strong interaction between graphene layer and Fano resonance in terahertz metamaterials. J. Phys. D Appl. Phys. 2017, 50, 195101. [CrossRef] 